\title{
New Era of Curative Therapy for Spinal Muscular Atrophy in Children; Do We Need Implementation of Universal Newborn Screening for Spinal Muscular Atrophy?
}

\author{
Nidheesh Cheeyancheri Chencheri ${ }^{1 *}$, Bhavna Gupta ${ }^{2}$ \\ ${ }^{1}$ Department of Paediatric Neurology, Al Jalila children's specialty hospital Dubai, United Arab Emirates \\ ${ }^{2}$ Department of Paediatrics, Al Qassimi Women and Children's hospital Shrajah, United Arab Emirates
}

\section{*Corresponding Author}

Nidheesh Cheeyancheri Chencheri

\section{Article History}

Received: 25.01.2021

Accepted: 09.02.2021

Published: 14.02 .2021

\section{Short communication/Letter to the editor}

Spinal muscular atrophy (SMA) is an autosomal recessive neuromuscular disease characterized by degeneration of alpha motor neurons in the spinal cord, resulting in progressive proximal muscle weakness and paralysis. SMA is the second most common fatal autosomal recessive disorder after cystic fibrosis, with an estimated incidence of 1 in 6,000 to 1 in 10,000 live births, with a carrier frequency of 1/40-1/60 [1]. The disease was first described in the 1890s by Werdnig and by Hoffmann [2]. This disease is characterized by generalized muscle weakness and atrophy predominating in proximal limb muscles, and phenotype is classified into four grades of severity (SMA I, SMAII, SMAIII, SMA IV) based on age of onset and motor function achieved [3]. This disease is caused by homozygous mutations of the survival motor neuron 1 (SMN1) gene, and the diagnostic test demonstrates in most patients the homozygous deletion of the SMN1 gene, generally showing the absence of SMN1 exon 7 [4]. The test achieves up to $95 \%$ sensitivity and nearly $100 \%$ specificity.

Till recently spinal muscular atrophy especially type 1 was a fatal disease. Most of the severe type of SMA dies or end up in permanent ventilation by 2 years of age. There is a new ray of hope for parents and scientific community when the much awaited genetic treatment was launched. First came Nusinersen (brand name Spinraza ${ }^{\circledR}$ ), which was FDA approved in December 2016. It works by boosting the efforts of SMN2 so it makes more SMN protein. It is given as intrathecal injection for patients of all ages. After four initial loading doses over two months, patients must continue receiving a dose every four months for life. Then a gene replacement therapy for SMA, onasemnogene abeparvovec-xioi (brand name Zolgensma), was approved by the FDA in May 2019, limited to patients under the age of two. Corrected copies of the SMN gene are delivered by a one-time intravenous infusion. These modified genes live in the cell nucleus and make SMN protein, thus sustaining the motor neurons [5]. This has completely changed the natural history of SMA. The studies clearly showed that early identification and the treatment prevent the death of neurons and hence better outcome. So "time is neurons". There comes the importance of identifying SMA by new born screening (NBS).

Newborn screening seems to be the best solution to guarantee early diagnosis of SMA. Neonatal screening tests are mass screening tests covering all newborns, enabling the detection of diseases that, if untreated, lead to serious developmental disorders and even death. The criteria for including the disease in neonatal screening were developed by Wilson and Junger in 1968 [6]. The basic criteria for introducing screening tests are economic, taking into account the incidence of disease in the population and the availability of treatment and care. Currently, with the development of medicine and diagnostic techniques, this approach is being revised. The economic factor is important but no longer the main criterion for introducing newborn screening tests. SMA meets all screening criteria according to Wilson and Junger. An important element of these criteria, apart from the severity and frequency of the disease and the effectiveness of early therapy, is the availability of a sensitive and relatively inexpensive diagnostic test. The situation of SMA is rare in clinical genetics, whereby the genetic basis of the disease is extremely homogeneous. In approximately 95-98\% of SMA patients, the disease is caused by bilateral loss of the SMN1 gene. This has enabled the development of very sensitive (95-98\%), specific (100\%) and relatively inexpensive genetic tests for SMA, which can be used on a mass scale and implemented for screening.

Copyright (C) 2021 The Author(s): This is an open-access article distributed under the terms of the Creative Commons Attribution 4.0 International License (CC BY-NC 4.0) which permits unrestricted use, distribution, and reproduction in any medium for non-commercial use provided the original author and source are credited. 
The emergence of real prospects for the treatment of SMA a few years ago started discussions on the implementation of neonatal screening for SMA. The systematic review of literature showed few studies looking at the new-born screening of SMA. A German pilot project study was conducted to assess the impact of early detection of SMA by newborn screening (NBS) on the clinical course of the disease [7]. Screening was performed in two federal states of Germany. 165,525 children were screened. 22 cases of SMA were identified, meaning an incidence rate of 1:7524. 10 patients with 2 or 3 SMN2 copies were treated with Nusinersen, starting between 15-39 days after birth, in 7/10 patients before onset of symptoms. Presymptomatically treated patients (age at last examination: 1-12 months, median 8 months) showed no muscle weakness by the age of one month to one year. Another study was conducted in three hospitals in New York and it allowed the identification of SMA in 1 of 3,826 newborns tested [8]. Currently, the US is the first country to gradually implement the NBS toward SMA for the entire population.

The current situation of neonatal screening for SMA is very promising. Pilot studies are underway in some countries, while in others, decisions or negotiations have already been made to add SMA to national screening programs. Unfortunately, the cost of treatment is exorbitantly high and only affluent nations can afford it at present.

\section{CONCLUSION}

Newborn screening can detect patients affected by SMA before symptom onset and enable early therapeutic intervention. Newborn screening for SMA should be introduced in all countries where therapy is available. Given the homogeneity of the genetic background of the disease and well-developed screening programs in most countries, the introduction of universal testing of new-borns for SMA does not appear difficult.

\section{REFERENCE}

1. D'Amico, A., Mercuri, E., Tiziano, F. D., \& Bertini, E. (2011). Spinal muscular atrophy. Orphanet journal of rare diseases, 6(1), 1-10.

2. Dubowitz, V. (2009). Ramblings in the history of spinal muscular atrophy. Neuromuscular disorders, 19(1), 69-73.

3. Crawford, T. O., \& Pardo, C. A. (1996). The neurobiology of childhood spinal muscular atrophy. Neurobiology of disease, 3(2), 97-110.

4. Gendron, N. H., \& MacKenzie, A. E. (1999). Spinal muscular atrophy: molecular pathophysiology. Current opinion in neurology, 12(2), 137-142.

5. Mercuri, E., Pera, M. C., Scoto, M., Finkel, R., \& Muntoni, F. (2020). Spinal muscular atrophy-Insights and challenges in the treatment era. Nature Reviews Neurology, 16(12), 706-715.

6. Wilson, J. M., \& Junger, G. (1968). Principles and practice of screening for disease. Genewa: WHO.

7. Vill, K., Kölbel, H., Schwartz, O., Blaschek, A., Olgemöller, B., Harms, E., ... \& Müller-Felber, W. (2019). One year of newborn screening for SMA-Results of a German pilot project. Journal of neuromuscular diseases, 6(4), 503-515.

8. Kraszewski, J. N., Kay, D. M., Stevens, C. F., Koval, C., Haser, B., Ortiz, V., ... \& Chung, W. K. (2018). Pilot study of population-based newborn screening for spinal muscular atrophy in New York state. Genetics in Medicine, 20(6), 608-613.

CITATION: Nidheesh Cheeyancheri Chencheri \& Bhavna Gupta (2021). New Era of Curative Therapy for Spinal Muscular Atrophy in Children Do We Need Implementation of Universal Newborn Screening for Spinal Muscular Atrophy?. South Asian Res J Med Sci, 3(1): 14-15. 